\title{
DESEMPENHO DE VÁLVULAS ANTIGOTAS E MICROASPERSORES
}

\author{
DELVIO SANDRI ${ }^{1}$, KADY S. E. BESSA ${ }^{2}$, MÁRCIO MESQUITA ${ }^{3}$, \\ LAZARO C. R. A. PRADO ${ }^{4}$
}

\begin{abstract}
RESUMO: Nos últimos anos, foram introduzidos novos modelos de dispositivos para cultivo em ambiente protegido, como as válvulas antigotas e microaspersores, necessitando-se avaliar o desempenho dos mesmos. Assim, o objetivo deste trabalho foi avaliar o desempenho hidráulico de válvulas antigotas novas e usadas, sob baixa e alta pressão, em microaspersores do grupo modular e modelo Hadar. O CVf e CVq do conjunto microaspersor e válvula antigotas de baixa pressão e somente o microaspersor modular foram classificados como ruins ou inaceitáveis e a válvula antigotas modular de alta pressão nova como inaceitável. A válvula Hadar apresentou o melhor desempenho para todas as pressões de serviço. As válvulas avaliadas abriram em pressões inferiores às indicadas pelos seus fabricantes. O tempo de uso interferiu no desempenho das válvulas antigotas modular de baixa pressão, sendo o modelo novo o que apresentou menor índice de CVf para a pressão de abertura e fechamento.
\end{abstract}

PALAVRAS-CHAVE: equação vazão x pressão, microaspersor, estufa, irrigação.

\section{PERFORMANCE OF LEAK-PREVENTING DEVICE AND MICROSPRINKLER}

ABSTRACT: In the last years new models of devices were introduced for greenhouse cultivation, as the leak-preventing device and microsprinkler, it is needed to evaluate their performance. So the objective of this work was to evaluate the hydraulic performance of new and used leak-preventing device, under low and high pressure in microsprinkler of modular group and Hadar model. The CVf and $\mathrm{CVq}$ of the microsprinkler and the leak-preventing device of low pressure only the microsprinkler of modular group were classified as bad or unacceptable and the new high pressure as unacceptable. The leak-preventing device Hadar presented the best performance for all the performances. The leak-preventing device opened in low pressures as indicated by their manufacturers. The long time using interfered in the performance of the leak-preventing device of low pressure modular group, being the new model which presented smaller values of CVf for the opening and closing pressure.

KEYWORDS: discharge x pressure equation, microsprinklers, green house, irrigation.

\section{INTRODUÇÃO}

Com o aumento da área irrigada, houve uma expansão do mercado de dispositivos para atenderem a essa demanda, surgindo novas empresas e novos produtos, implicando a necessidade de determinação de suas características estruturais e de análise de seu desempenho, bem como estabelecer novos parâmetros para torná-los mais eficazes e de menor custo (MAZZER et al., 2003; SILVA \& SILVA, 2003). Segundo SIQUEIRA \& BARROS JÚNIOR (2003), a principal finalidade de ensaios de equipamentos utilizados em sistemas de irrigação localizada consiste na determinação e avaliação de suas características hidráulicas. São muitos os fatores que influenciam ou afetam a eficiência de aplicação d'água, destacando-se: variação da vazão devido ao processo de fabricação; expoente da pressão na equação de vazão do emissor e estabilidade da vazão em função da pressão;

\footnotetext{
${ }^{1}$ Prof. DE V, Faculdade de Engenharia Agrícola, UnUCET, UEG, Caixa Postal 459, Anápolis - GO, sandri@ ueg.br.

${ }^{2}$ Eng ${ }^{\mathrm{o}}$ Agrícola, UnUCET, UEG, Anápolis - GO, kseb40@ hotmail.com.

${ }_{3}^{3}$ Doutorando em Engenharia Agrícola, FEAGRI, UNICAMP, Campinas - SP, marcio.mesquita@ feagri.unicamp.br.

${ }^{4}$ Mestrando, IESA-Geografia, UFG, Goiânia - GO, lazaroagricola@gmail.com.

Recebido pelo Conselho Editorial em: 18-6-2008

Aprovado pelo Conselho Editorial em: 15-4-2010
} 
variação da pressão de funcionamento; perda de carga em razão da inserção do emissor na linha lateral e suscetibilidade a entupimento por sedimentação e precipitação de sais.

Para se obterem melhores resultados de determinada cultura, o desempenho hidráulico deve ser associado a outros fatores, como o elevado consumo de água e às restrições de disponibilidade da mesma, bem como a possíveis riscos de degradação do sistema solo-água-planta (SANTIAGO et al., 2004) e fatores ambientais, como temperatura e umidade do ar, que quando adequadamente medidos, podem auxiliar na determinação da necessidade ou não da utilização de tecnologias mais avançadas (COSTA et al., 2004).

Em ambiente protegido, após a interrupção da irrigação por microaspersão, as tubulações permanecem cheias de água, que tende a se deslocar para pontos mais baixos, drenando pelos microaspersores ou sprays, favorecendo o surgimento de doenças pela manutenção de condições adequadas de umidade para proliferação de microrganismos, especialmente sob os emissores, além de provocar o arranquio de plantas. Assim, principalmente na formação de mudas de hortaliças, são utilizadas válvulas antigotas que, com o tempo de uso, podem sofrer danos, alterando as pressões de abertura e fechamento e comprometendo seu desempenho. São de fácil funcionamento, manutenção e instalação. No entanto, as informações quanto ao desempenho hidráulico é bastante limitada, e na maioria das vezes, restringe-se a dados de pressão de abertura e fechamento fornecidos pelos seus fabricantes. Não são encontrados na literatura especializada trabalhos para avaliação do seu desempenho após determinado tempo de uso. Utilizadas acopladas a microaspersores, nebulizadores e sprays para irrigação ou controle das condições climáticas em ambiente protegido ou em pulverização agrícola, a válvula antigotas é um dispositivo que interrompe a passagem de água, quando o sistema de bombeamento é desligado e a pressão diminui. A abertura do diafragma da válvula ocorre quando uma determinada pressão é alcançada e fecha-se ao reduzir a pressão, e a válvula volta ao seu estado de repouso, obstruindo a passagem de água.

A válvula antigotas evita desperdício de água, mantendo as tubulações cheias de água, iniciando a irrigação ao mesmo tempo em todos os pontos, por criar pulsos mais curtos e eficientes (SAMPAIO, 2001; SAPIR \& SNEH, 2002). Nesse sentido, este trabalho teve o objetivo de avaliar o desempenho hidráulico de válvulas antigotas novas e usadas, sob baixa e alta pressão, em microaspersores do grupo modular e modelo Hadar.

\section{MATERIAL E MÉTODOS}

O trabalho foi realizado no Laboratório de Hidráulica da Unidade Universitária de Ciências Exatas e Tecnológicas da Universidade Estadual de Goiás, Anápolis - GO.

Foram avaliadas 80 válvulas antigotas, escolhidas aleatoriamente dentro de um lote fornecido pelo fabricante, sendo 20 amostras para cada modelo: modular de baixa pressão nova (Figura 1A) e usada; modular nova de alta pressão roscável (Figura 1B) e Hadar nova de baixa pressão blindada (Figura 1C). As válvulas modulares foram testadas com o microaspersor modular novo, vazão de $120 \mathrm{~L} \mathrm{~h}^{-1}$ (Figura 1D) e a válvula Hadar com o microaspersor Hadar, vazão de $41 \mathrm{~L} \mathrm{~h}^{-1}$ (Figura 1E).
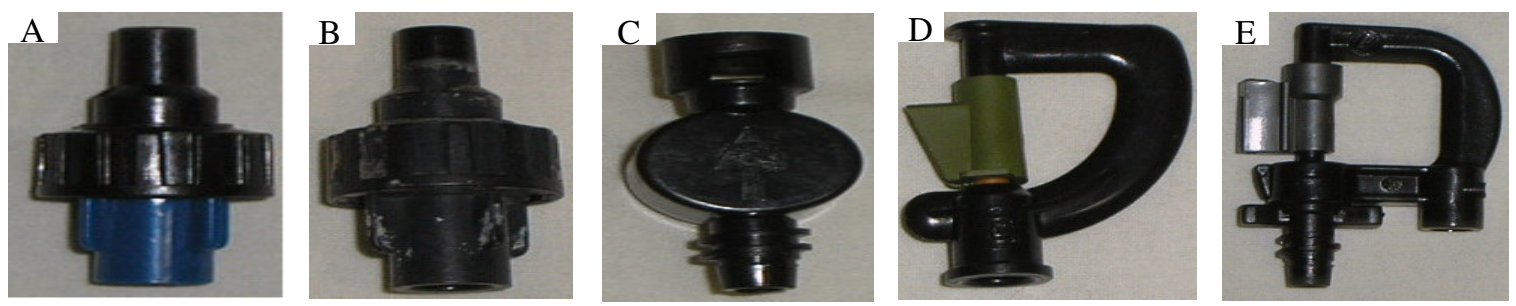

FIGURA 1. Válvulas antigotas do grupo modular de alta (A) e baixa (B) pressão, modelo Hadar de baixa pressão (C) e microaspersores do grupo modular (D) e modelo Hadar (E). Leakpreventing device of modulate group high pressure $(\mathrm{A})$ and low pressure (B), Hadar model low pressure (C) and microsprinkler modular group (D) and Hadar (E). 
As válvulas usadas tinham cerca de cinco anos de uso, retiradas de uma casa de vegetação onde se produziram mudas de hortaliças. De acordo com o fabricante, a válvula do grupo modular de alta pressão abre em torno de $350 \mathrm{kPa}$ e fecha a $200 \mathrm{kPa}$, enquanto a de baixa pressão abre a $200 \mathrm{kPa}$ e fecha a $100 \mathrm{kPa}$. A válvula Hadar de baixa pressão abre a $130 \mathrm{kPa}$ e fecha a $90 \mathrm{kPa}$.

Foi utilizado o mesmo modelo de microaspersor novo para ensaio das válvulas modulares de baixa pressão nova e usada e alta pressão, de forma que possíveis alterações fossem devidas somente à válvula antigotas. Foram realizados ensaios com o conjunto válvula antigotas e microaspersor e somente do microaspersor, utilizando uma bancada de ensaio composta por um conjunto motobomba de $3 \mathrm{cv}$, um reservatório de $0,50 \mathrm{~m}^{3}$ e tubulações de PVC rígido e mangueira de polietileno de 3/4', formando um circuito hidráulico fechado. Sobre o reservatório, instalou-se uma mangueira de polietileno, permitindo o ensaio de 10 microaspersores ao mesmo tempo, distantes 0,25 m entre cada saída. Sob cada microaspersor, acomodou-se um recipiente de 10,0 L, apoiado sobre uma tábua, que permitia seu deslocamento manualmente, facilitando a coleta da vazão (Figura 2). A pressão foi medida com um manômetro de Bourdon com glicerina, precisão de $2 \mathrm{kPa}$ e a pressão ajustada com um registro de agulha.

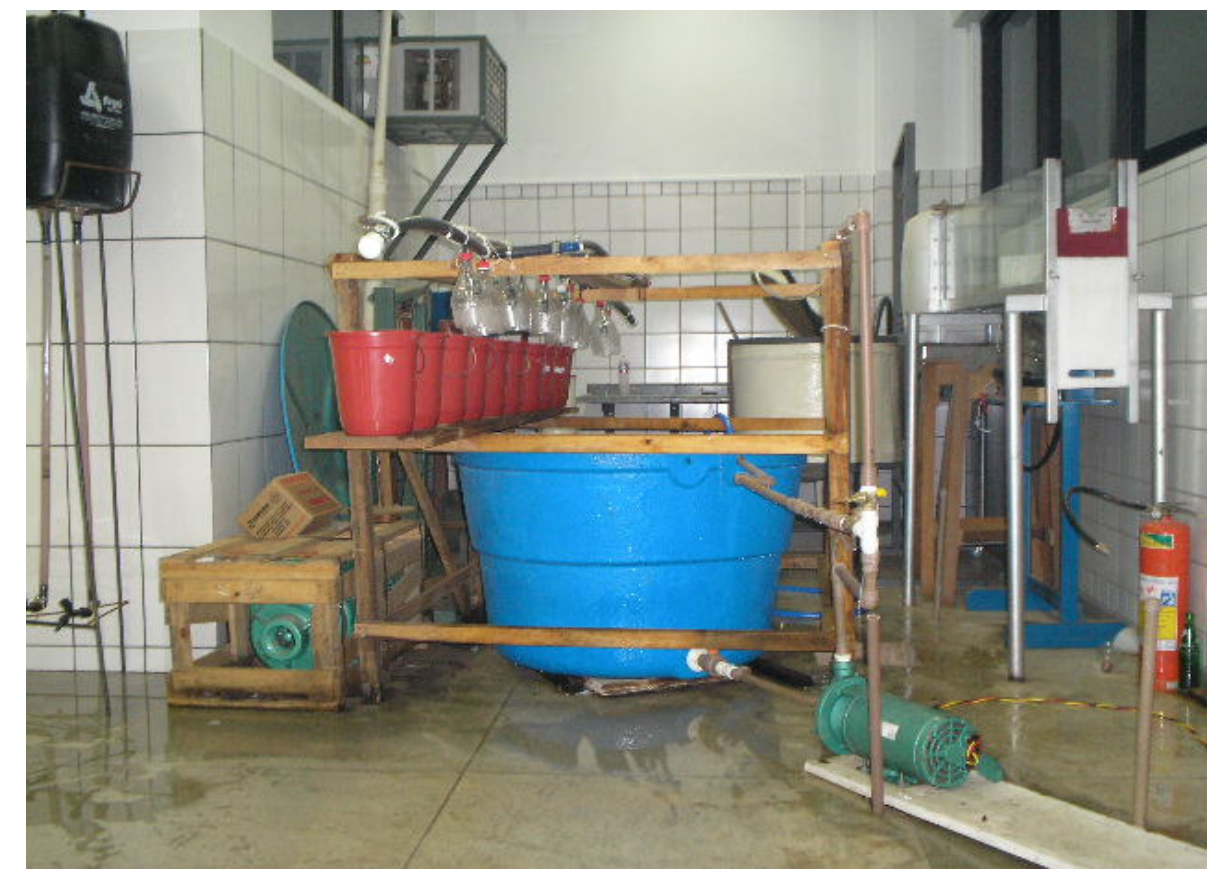

FIGURA 2. Módulo de ensaio dos microaspersores para a obtenção da equação vazão x pressão. Module test of microsprinkler to obtain the discharge $x$ pressure relation.

Para o conjunto microaspersor e válvula antigotas de baixa pressão, utilizaram-se das pressões 150; 200; 250; 300 e $350 \mathrm{kPa}$ e, para o de alta pressão, de 340; 360; 380; 400 e $420 \mathrm{kPa}$. A pressão mínima utilizada para a válvula de alta pressão deve-se à sua característica de não abrir sob pressões menores. A vazão foi medida pelo método gravimétrico, com auxílio de uma balança eletrônica digital, com precisão de $0,01 \mathrm{~g}$. O tempo de coleta foi de três minutos, medidos com um cronômetro digital, com três repetições.

Para a determinação das pressões de abertura e fechamento das 20 válvulas antigotas de cada modelo, utilizou-se do mesmo módulo de ensaio. Porém, substituiu-se a tubulação com dez saídas por outra de PVC de 3/4', com apenas uma saída, para avaliação de uma única válvula por vez. Para medida da pressão, utilizou-se de um manômetro de mercúrio em " $U$ ". O procedimento consistiu em acionar o sistema de bombeamento, abrindo-se lentamente o registro de agulha até que começasse sair água pelo microaspersor (primeiras gotas), anotando-se a pressão como sendo a de abertura da válvula. Prosseguiu-se o ensaio com a abertura do registro até se atingir a pressão de aproximadamente $230 \mathrm{kPa}$ para as válvulas de baixa pressão e $310 \mathrm{kPa}$ para as válvulas de alta 
pressão para, posteriormente, proceder ao fechamento do registro até a válvula antigotas interromper totalmente o fluxo de água, anotando-se a pressão como sendo a de fechamento. Repetiu-se este procedimento três vezes para cada válvula antigotas, calculando-se a média da pressão de abertura e fechamento.

A curva característica vazão x pressão, de acordo com BERNARDO et al. (2005), pode ser representada pela eq.(1):

$$
\mathrm{q}=\mathrm{Kh} \mathrm{x}^{\mathrm{x}}
$$

em que,

q - vazão do emissor, $\mathrm{L} \mathrm{h}^{-1}$;

$\mathrm{K}$ - coeficiente de descarga (constante, característico de cada emissor);

$\mathrm{h}$ - pressão na entrada, $\mathrm{kPa}$, e

$\mathrm{x}$ - expoente de descarga (característico de cada emissor, que representa o tipo de fluxo).

O coeficiente de variação de fabricação - CVf para peças novas e o coeficiente de variação de vazão - CVq para peças usadas foram determinados pela Equação 2 (KELLER \& KARMELI, 1984). O CVf é calculado para equipamentos novos, onde se analisa a correspondência com a informada pelo fabricante para cada pressão de serviço. $\mathrm{O} C \mathrm{Cq}$ é determinado para equipamentos usados, para verificar possíveis alteração das características originais devido ao tempo de uso.

$$
\text { CVf ou } C V q=\left[\left(\sum \mathrm{q}_{\mathrm{i}}-\mathrm{nq}_{\mathrm{m}}{ }^{2}\right) /(\mathrm{n}-1)\right]^{0,5} / \mathrm{q}_{\mathrm{m}}
$$

em que,

CVf - coeficiente de variação de fabricação, decimal;

$\mathrm{CVq}$ - coeficiente de variação de vazão, decimal;

$\mathrm{q}_{\mathrm{i}}$ - vazões individuais de cada emissor, $\mathrm{L} \mathrm{h}^{-1}$;

$\mathrm{q}_{\mathrm{m}}$ - vazão média dos emissores, $\mathrm{L} \mathrm{h}^{-1}$, e

n - número de emissores no lote de amostragem avaliados.

A classificação do coeficiente de variação de fabricação e de vazão das válvulas e dos microaspersores seguiu a ASAE EP 405.1 (1994) e o Projeto de norma 12: 02. 08-22 da ABNT (1986). A ASAE (1994) classifica os emissores, quanto à uniformidade do CVf, da seguinte maneira: abaixo de $5 \%$ em excelente; entre $5 \%$ e $7 \%$ em média; entre $7 \%$ e $11 \%$ em marginal; entre $11 \%$ e $15 \%$ em ruim e acima $15 \%$ como inaceitável, enquanto para a ABNT (1986), valores inferiores a $10 \%$ é boa; entre $10 \%$ e $20 \%$ é média; entre $20 \%$ e $30 \%$ é marginal e superior a $30 \%$ é inaceitável.

\section{RESULTADOS E DISCUSSÃO}

Na Tabela 1, estão apresentados os resultados de desempenho do microaspersor do grupo modular novo, funcionando sem a válvula antigotas. Observa-se que o desvio-padrão variou entre $11,17 \mathrm{~L} \mathrm{~h}^{-1}$ e $18,06 \mathrm{~L} \mathrm{~h}^{-1}$, com valor médio de 14,59 $\mathrm{L} \mathrm{h}^{-1}$, aumentando com a elevação da pressão. O valores de CVf variaram entre $11,88 \%$ e $12,28 \%$, com valor médio de $11,88 \%$, com maior valor para a pressão mais elevada. O CVf foi classificado como médio, de acordo com a ABNT (1986), e ruim, pela ASAE (1994), para todas as pressões. As vazões apresentaram grande variação entre as amostras avaliadas, podendo ser atribuída ao processo de fabricação.

A vazão obtida nesse experimento foi inferior à fornecida pelo catálogo do fabricante, sendo de $10,49 \%$ para a pressão de $150 \mathrm{kPa} ; 8,84 \%$ na de $200 \mathrm{kPa} ; 8,24 \%$ na de $250 \mathrm{kPa} ; 8,45 \%$ na de $300 \mathrm{kPa}$, e 7,53\% na de $350 \mathrm{kPa}$.

O desempenho entre modelos e marcas de microaspersores é bastante variável, como mostra o trabalho de MATOS \& RAGOSO (1997), que avaliaram os microaspersor Carborundum, modelo MSIII, vazões nominal de $43 \mathrm{~L} \mathrm{~h}^{-1}$, com bocal verde-claro e rosa. O modelo com bocal verde-claro apresentou para a pressão de $150 \mathrm{kPa}, \mathrm{CVf}$ de $6,9 \%$, e para o modelo de bocal rosa, o CVf foi de 
6,8\% e desvio-padrão de 2,92 $\mathrm{L} \mathrm{h}^{-1}$ e, com classificação média, segundo a ASAE (1994), valores estes inferiores aos observados neste trabalho para o microaspersor do grupo modular novo, bocal laranja.

TABELA 1. Vazão média, desvio-padrão, coeficiente de variação de fabricação - CVf e classificação segundo ASAE (1994) e ABNT (1986), do microaspersor do grupo modular novo, para diferentes pressões. Average discharge rate, standard deviation, manufacturing coefficient variation - CVf and classification according to ASAE (1994) and ABNT (1986) of the microsprinkler of the new modular group for different pressures.

\begin{tabular}{cccccc}
\hline Pressão & Vazão Média & Desvio-Padrão & CVf & \multicolumn{2}{c}{ Classificação } \\
\cline { 5 - 6 }$(\mathrm{kPa})$ & $\left(\mathrm{L} \mathrm{h}^{-1}\right)$ & $\left(\mathrm{L} \mathrm{h}^{-1}\right)$ & $(\%)$ & ASAE (1992) & ABNT (1986) \\
\hline 150 & 93,99 & 11,17 & 11,98 & Ruim & Médio \\
200 & 109,40 & 13,01 & 11,89 & Ruim & Médio \\
250 & 122,96 & 14,66 & 11,92 & Ruim & Médio \\
300 & 134,58 & 16,06 & 11,93 & Ruim & Médio \\
350 & 147,04 & 18,06 & 12,28 & Ruim & Médio \\
\hline Média & - & 14,59 & 11,88 & - & - \\
\hline
\end{tabular}

O microaspersor do grupo modular novo, funcionando com válvula antigotas modular nova de baixa pressão, apresentou aumento no valor do desvio-padrão e CVf em relação a somente o microaspersor. $\mathrm{O}$ valor médio do desvio-padrão passou para 14,83\%, variando de $11,35 \%$ a $17,92 \%$, e o CVf médio mudou para $12,36 \%$, variando de $12,30 \%$ a $12,40 \%$, demonstrando efeito negativo do uso da válvula antigotas sobre o desempenho do conjunto (Tabela 2). $\mathrm{O}$ aumento da pressão de serviço também elevou o desvio-padrão, devendo-se provavelmente a alteração da dilatação do diafragma com o tempo de uso. A classificação do CVf não alterou quando se avaliou o conjunto microaspersor mais válvula antigotas nova em relação a somente o microaspersor, permanecendo ruim segundo a ASAE (1994) e médio pela ABNT (1986).

Utilizando o microaspersor novo, porém com válvula antigotas modular usada de baixa pressão, o desvio-padrão médio foi de $18,73 \mathrm{~L} \mathrm{~h}^{-1}$ e $\mathrm{CVq}$ de $25,48 \%$, muito superior ao conjunto microaspersor mais válvula antigotas novos (Tabela 2). Esse comportamento deve-se à grande variação entre as válvulas, especialmente na pressão de $150 \mathrm{kPa}$, reduzindo a vazão em 105,89\%, passando de $92,26 \mathrm{~L} \mathrm{~h}^{-1}$ para válvulas novas á 44,81 $\mathrm{L} \mathrm{h}^{-1}$ para a válvula usada, elevando o desvio-padrão a $35,24 \mathrm{~L} \mathrm{~h}^{-1}$ e o $\mathrm{CVq}$ á $80,69 \%$. Isso se deve provavelmente ao ressecamento do diafragma, abrindo numa pressão superior às válvulas novas e reduzindo a vazão do microaspersor. Considerando a válvula antigotas usada mais o microaspersor, houve um decréscimo na vazão de $1,73 \mathrm{~L} \mathrm{~h}^{-1}$ na pressão de $150 \mathrm{kPa}$ e redução de $0,84 \mathrm{~L} \mathrm{~h}^{-1}$ na pressão de $300 \mathrm{kPa}$, em relação a somente o microaspesor.

A redução da vazão deve-se à perda de carga e demonstram que as válvulas usadas diminuíram sua elasticidade, onde pressões menores, mesmo dentro da faixa indicada pelo fabricante, não apresentam resultados satisfatórios, por dificultar a abertura do diafragma, provavelmente por ressecamento da borracha do diafragma. Para as demais pressões, os valores de CVq alteram-se pouco em relação aos de CVf, embora melhores resultados tenham sido obtidos na maior pressão, alterando a classificação para marginal pela ASAE (1994), demonstrando ainda que válvulas antigotas do grupo modular apresentam melhor desempenho para pressões acima de $200 \mathrm{kPa}$. 
TABELA 2. Vazão média, desvio-padrão, coeficiente de variação de fabricação - CVf e de vazão CVq e classificação segundo ASAE (1994) e ABNT (1986), do conjunto microaspersor e válvula antigotas modular nova e usada de baixa pressão, para diferentes pressões. Average discharge rate, standard deviation, manufacturing coefficient variation - CVf and flow - CVq and classification according to ASAE (1994) and ABNT (1986) of the set microsprinkler and leak-preventing device new and used of the modulate group of low pressure for different pressures.

\begin{tabular}{|c|c|c|c|c|c|c|c|c|c|c|}
\hline \multirow{3}{*}{$\begin{array}{c}\text { Pressão } \\
(\mathrm{kPa})\end{array}$} & \multirow{2}{*}{\multicolumn{2}{|c|}{$\begin{array}{l}\text { Vazão Média } \\
\left(\mathrm{L} \mathrm{h}^{-1}\right)\end{array}$}} & \multirow{2}{*}{\multicolumn{2}{|c|}{$\begin{array}{c}\text { Desvio-Padrão } \\
\left(\mathrm{L} \mathrm{h}^{-1}\right)\end{array}$}} & \multirow{3}{*}{$\begin{array}{c}\text { CVf } \\
(\%)\end{array}$} & \multirow{3}{*}{$\begin{array}{c}\text { CVq } \\
(\%) \\
\text { usada }\end{array}$} & \multicolumn{4}{|c|}{ Classificação } \\
\hline & & & & & & & \multicolumn{2}{|c|}{ ASAE (1992) } & \multicolumn{2}{|c|}{ ABNT (1986) } \\
\hline & nova & Usada & nor & usada & & & nova & usada & va & Usada \\
\hline 150 & 26 & & & & 12,30 & 80,69 & $\mathrm{~m}$ & eit & & \\
\hline 200 & 2 & $10^{4}$ & & & 36 & 12,08 & $\mathrm{~m}$ & Ruim & & \\
\hline 250 & 4 & 120 & & & 12,40 & 12 & $\mathrm{Ru}$ & Ruim & 10 & \\
\hline 300 & 14 & 13 & & & 12 , & 12 & & Ruim & & \\
\hline 350 & 5,20 & 44,34 & & & 12,34 & 10,33 & Ruim & Marginal & Médio & Médio \\
\hline Média & 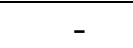 & - & 14,83 & 18,73 & 12,36 & 25,48 & 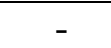 & 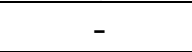 & 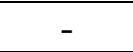 & 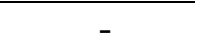 \\
\hline
\end{tabular}

O microaspersor do grupo modular novo, funcionando com válvula antigotas modular nova de alta pressão, apresentou desvio-padrão variando entre $75,88 \mathrm{~L} \mathrm{~h}^{-1}$ e $79,00 \mathrm{~L} \mathrm{~h}^{-1}$ e valor médio de $77,88 \%$, sendo considerado muito elevado. O CVf variou entre 57,04\% e 113,68\% com valor médio de 85,28\%, classificado como inaceitável pela ABNT (1986) e ASAE (1994) (Tabela 3). O CVf diminuiu com o aumento da pressão, embora nas menores pressões, a abertura das válvulas foi muito irregular, onde, enquanto algumas estavam liberando água, outras permaneciam totalmente fechadas, demonstrando a grande irregularidade no processo de fabricação do diafragma da válvula.

TABELA 3. Vazão média, desvio-padrão, coeficiente de variação de fabricação - CVf e classificação segundo ASAE (1994) e ABNT (1986), do conjunto microaspersor e válvula antigotas modular nova de alta pressão, para diferentes pressões. Average discharge rate, standard deviation, manufacturing coefficient variation - CVf and classification according to ASAE (1994) and ABNT (1986) of the set microsprinkler and leak-preventing device new of the modulate group of high pressure for different pressures.

\begin{tabular}{|c|c|c|c|c|c|}
\hline \multirow{2}{*}{$\begin{array}{c}\text { Pressão } \\
(\mathrm{kPa})\end{array}$} & \multirow{2}{*}{$\begin{array}{l}\text { Vazão Média } \\
\left(\mathrm{L} \mathrm{h}^{-1}\right)\end{array}$} & \multirow{2}{*}{$\begin{array}{c}\text { Desvio-Padrão } \\
\left(\mathrm{L} \mathrm{h}^{-1}\right)\end{array}$} & \multirow{2}{*}{$\begin{array}{l}\text { CVf } \\
(\%)\end{array}$} & \multicolumn{2}{|c|}{ Classificação } \\
\hline & & & & ASAE (1992) & ABNT (1986) \\
\hline 340 & 68,33 & 77,68 & 113,68 & Inaceitável & Inaceitável \\
\hline 360 & 76,23 & 75,88 & 99,54 & Inaceitável & Inaceitável \\
\hline 380 & 87,32 & 79,00 & 90,47 & Inaceitável & Inaceitável \\
\hline 400 & 119,71 & 78,59 & 65,65 & Inaceitável & Inaceitável \\
\hline 420 & 137,15 & 78,24 & 57,04 & Inaceitável & Inaceitável \\
\hline Média & - & 77,88 & 85,28 & - & - \\
\hline
\end{tabular}

O microaspersor modelo Hadar 7110, funcionando sem válvula antigotas, apresentou desvio-padrão variando entre $2,17 \mathrm{~L} \mathrm{~h}^{-1}$ e $5,04 \mathrm{~L} \mathrm{~h}^{-1}$ e valor médio de $3,80 \mathrm{~L} \mathrm{~h}^{-1}$ e CVf entre 4,66\% e $12,00 \%$ e valor médio de $8,80 \%$ (Tabela 4). Valores esses, muito superiores aos obtidos por DANTAS NETO et al. (1997), avaliando somente o mesmo microaspersor, observando para a pressão de $200 \mathrm{kPa}$, desvio-padrão de $0,195 \mathrm{~L} \mathrm{~h}^{-1}$, CVf de $0,49 \%$ e vazão média de $39,8 \mathrm{~L} \mathrm{~h}^{-1}$, está pouco superior à obtida neste trabalho $\left(38,04 \mathrm{~L} \mathrm{~h}^{-1}\right)$. A diferença entre o resultado dos trabalhos deve-se à variação entre os lotes de produtos, ao processo de fabricação, como mudanças da linha de montagem, especialmente das máquinas extrusoras, qualidade da matéria-prima utilizada na fabricação, adoção de novas tecnologias pelo fabricante, dentre outros fatores, interferindo no desempenho hidráulico. 
A válvula antigotas de alta pressão instalada em conjunto com o microaspersor Hadar 7110, novos, apresentou desvio-padrão entre $2,77 \mathrm{~L} \mathrm{~h}^{-1}$ e $5,48 \mathrm{~L} \mathrm{~h}^{-1}$, com valor médio de $4,19 \mathrm{~L} \mathrm{~h}^{-1}$ e CVf entre $6,15 \%$ e $13,60 \%$, com valor médio de $10,10 \%$, maiores que os observados somente do microaspersor, demonstrando que a válvula antigotas reduz o desempenho hidráulico do conjunto, possivelmente, interferindo na uniformidade de distribuição de água. A classificação do CVf foi considerada marginal nas pressões de $150 ; 200$ e $350 \mathrm{kPa}$, ruim na de $250 \mathrm{kPa}$ e mediano na de $300 \mathrm{kPa}$, tanto somente para o microaspersor, como para a válvula antigotas mais o microaspersor, segundo a ASAE (1994), enquanto de acordo com a ABNT (1986), foi média para a pressão de $250 \mathrm{kPa}$ e boa nas demais pressões para o microaspersor e microaspersor mais válvula antigotas foi boa na pressão de $300 \mathrm{kPa}$ e média para as demais pressões.

TABELA 4. Vazão média, desvio-padrão, coeficiente de variação de fabricação - CVf e classificação segundo ASAE (1994) e ABNT (1986), do microaspersor modelo Hadar 7110, sem e com válvula antigotas novos, para diferentes pressões. Average discharge rate, standard deviation, manufacturing coefficient variation - CVf and classification according to ASAE (1994) and ABNT (1986) of microsprinkler Hadar model with and without leak-preventing device new for different pressures.

\begin{tabular}{|c|c|c|c|c|c|c|c|c|c|c|}
\hline \multirow{3}{*}{$\begin{array}{c}\text { Pressão } \\
(\mathrm{kPa})\end{array}$} & \multirow{2}{*}{\multicolumn{2}{|c|}{$\begin{array}{c}\text { Vazão Média } \\
\left(\mathrm{L} \mathrm{h}^{-1}\right)\end{array}$}} & \multirow{2}{*}{\multicolumn{2}{|c|}{$\begin{array}{c}\text { Desvio-Padrão } \\
\left(\mathrm{L} \mathrm{h}^{-1}\right)\end{array}$}} & \multirow{2}{*}{\multicolumn{2}{|c|}{$\begin{array}{l}\mathrm{CVf} \\
(\%)\end{array}$}} & \multicolumn{4}{|c|}{ Classificação } \\
\hline & & & & & & & \multicolumn{2}{|c|}{ ASAE (1992) } & \multicolumn{2}{|c|}{ ABNT (1986) } \\
\hline & sem & com & sem & com & sem & com & sem & com & sem & com \\
\hline 150 & 36,04 & 34,36 & 3, & 3,44 & 9 , & 10 , & Marginal & $\bar{M}$ & Boa & Média \\
\hline 200 & 39,41 & 38,04 & 3,47 & 3,82 & 8,80 & 10,04 & Marginal & Marginal & Boa & Média \\
\hline 250 & 41,98 & 40,06 & 5,04 & 5,45 & 12,00 & 13,60 & Ruim & Ruim & Média & Média \\
\hline 300 & 46,53 & 45,03 & 2,17 & 2,77 & 4,66 & 6,15 & Mediano & Mediano & Boa & Boa \\
\hline 350 & 2,50 & 51,10 & 5,04 & 5,48 & 9,60 & 10,72 & Marginal & Margi & Boa & Média \\
\hline Média & - & - & 3,80 & 4,19 & 8,80 & 10,10 & - & - & - & - \\
\hline
\end{tabular}

A vazão do microaspersor Hadar 7110 sem a válvula antigotas foi maior, comparado ao uso da válvula antigotas, sendo de 4,89\%, 3,60\%, 4,79\%,3,33\% e 2,74\%, para as pressões de $150 \mathrm{kPa}$, $200 \mathrm{kPa}, 250 \mathrm{kPa}, 300 \mathrm{kPa}$ e $350 \mathrm{kPa}$, respectivamente (Tabela 4), demonstrando que as duas maiores pressões resultaram em melhores índices, que se deveu à maior estabilidade na dilatação do diafragma da válvula.

A curva característica do microaspersor modular com válvula antigota nova de alta pressão, bocal laranja apresentou melhor ajuste com a equação polinomial quadrática do que com a equação potencial. Isso se deve às válvulas antigotas modulares de alta pressão apresentarem uma variação de pressão de abertura e fechamento elevadas entre as amostras analisadas, apresentando melhores índices de CVf quando se aumentou a pressão. Devido à limitação da estação de bombeamento, não foi possível avaliar para pressões maiores $(>350 \mathrm{kPa})$, como as indicadas para sprays e nebulizadores, onde provavelmente os resultados seriam melhores (Tabela 5).

TABELA 5. Equação característica vazão $x$ pressão e coeficiente de determinação dos microaspersores e válvulas avaliadas. Discharge x pressure equation and coefficient of determination of microsprinkler and evaluated valves.

\begin{tabular}{lcc}
\hline Microaspersor e Válvula & Equação Vazão x Pressão & $\mathrm{R}^{2}$ \\
\hline Modular sem válvula antigotas & $\mathrm{q}=6,7587 \mathrm{~h}^{0,5253}$ & 0,9999 \\
Modular mais válvula antigotas nova de baixa pressão & $\mathrm{q}=6,3526 \mathrm{~h}^{0,5341}$ & 0,9999 \\
Modular mais válvula antigotas usada de baixa pressão & $\mathrm{q}=0,5054 \mathrm{~h}^{0,9751}$ & 0,8410 \\
Modular mais válvula antigotas nova de alta pressão & $\mathrm{q}=789,0786-4,574 \mathrm{~h}+0,0072 \mathrm{~h}^{2}$ & 0,9783 \\
Hadar 7110 sem válvula antigotas de baixa pressão & $\mathrm{q}=3,7023 \mathrm{~h}^{0,4472}$ & 0,9499 \\
Hadar 7110 mais válvula antigotas de baixa pressão & $\mathrm{q}=3,1433 \mathrm{~h}^{0,4702}$ & 0,9430 \\
\hline
\end{tabular}


O microaspersro Hadar 7110, sem válvula antigotas de baixa pressão, apresentou a equação, $\mathrm{q}=3,7023 \mathrm{~h}^{0,4472}$ e $\mathrm{R}^{2}=0,9499$, ajuste inferior ao obtido por DANTAS NETO et al. (1997), para este mesmo microaspersor, obtendo: $\mathrm{q}=2,4854 \mathrm{~h}^{0,5235}$ e $\mathrm{R}^{2}=0,9999$.

Com exceção ao conjunto microaspersor modular e válvula antigotas usada de baixa pressão, em que o expoente "x" da equação foi próximo a 1,0 e com coeficiente de determinação de 0,8410 , ou seja, menor em relação às demais equações obtidas, devido à dificuldade de abertura do diafragma para a pressão de $150 \mathrm{kPa}$, comprometendo o ajuste da equação do tipo potencial e também ao conjunto microaspersor modular e válvula antigotas nova de alta pressão, onde a equação que apresentou melhor ajuste foi a do tipo polinomial quadrática, as demais equações apresentaram expoente " $x$ " da equação característica próximo a 0,50.

Segundo KELLER \& KARMELI (1984), este valor caracteriza o emissor como sendo de fluxo turbulento, significando que as mudanças na vazão se relacionam com a raiz quadrada da pressão e, consequentemente, uma variação na pressão da ordem de $20 \%$ permite a variação da vazão dos emissores em $\pm 10 \%$ em relação ao valor desejado.

O modelo de válvula antigotas do grupo modular de baixa pressão nova apresentou pressão de abertura média de $120,5 \mathrm{kPa}$ e CVf de 5,68\%, considerado segundo a ASAE (1994) como mediano e segundo a ABNT (1986) como bom. O CVf de fechamento foi de 4,82\%, considerado excelente segundo ASAE (1994) e bom para a ABNT (1986), com pressão média de 109,5 kPa (Tabela 6), diferindo grandemente dos dados fornecidos pelo fabricante, especialmente para a pressão de abertura. Essa foi a válvula que demorou mais tempo para obstruir a passagem de água, devido ao diafragma não ter elasticidade suficiente que permitisse a sua volta ao estado de repouso rapidamente.

A válvula antigotas do grupo modular de baixa pressão usada apresentou pressão de abertura média de $107,0 \mathrm{kPa}$, desvio-padrão de $27,6 \mathrm{kPa}$, com CVq de $25,78 \%$. Já na pressão de fechamento, apresentou média de $97,5 \mathrm{kPa}$ e desvio-padrão de 26,7 kPa com CVq de fechamento 27,42\%. Em ambos os casos, a classificação foi considerada inaceitável segundo ASAE (1994) e marginal pela ABNT (1986).

A pressão de abertura das válvulas antigotas modulares novas e usadas foram inferiores aos dados fornecidos pelo seu fabricante, enquanto para a pressão de fechamento foram maiores na válvula nova e menor na usada, porém com valores próximos aos valores de catálogo. As pressões médias de abertura das válvulas novas foram de $66,66 \%, 32,07 \%$ e 36,63\%, menores em relação aos dados fornecidos pelos seus fabricantes, para a modular de baixa pressão, de alta pressão e Hadar, respectivamente (Tabela 6).

A válvula antigotas modular de alta pressão nova apresentou pressão de abertura média de 265,44 kPa e CVf de 18,92\%; já a pressão de fechamento média foi de 254,94 kPa e CVf de $18,51 \%$, diferindo dos dados obtidos no catálogo do fabricante, que deveriam ser de $250 \mathrm{kPa}$ (abertura) e $200 \mathrm{kPa}$ (fechamento). A pressão de abertura foi de cerca de $10 \mathrm{kPa}$ maior em relação à de fechamento, enquanto e pelo seu fabricante é de $150 \mathrm{kPa}$. Em ambos os casos, a classificação foi considerada inaceitável pela a ASAE (1994) e médio pela ABNT (1986). Das 20 válvulas avaliadas, três delas (7; 9 e 17) apresentaram defeito de fabricação, com o diafragma solto dentro da estrutura (corpo) da válvula, não obstruindo a passagem da água, nem mesmo com o sistema despressurizado (Tabela 6).Ressalta-se a necessidade de descartar as válvulas que não impedem o gotejo mesmo após a despressurização do sistema hidráulico, como observado, uma vez que poderá favorecer o surgimento de doenças e distribuir-se facilmente por todo o ambiente de cultivo, comprometendo a produção. As válvulas antigotas avaliadas, tanto novas como as usadas, não apresentaram resultados satisfatórios, podendo interferir no desempenho do sistema de irrigação, uma vez que pressões de abertura maiores ou menores que as indicadas pelo fabricante comprometem o dimensionamento correto do sistema de bombeamento, e, por consequência, a vazão do sistema e lâmina de água aplicada. 
TABELA 6. Pressão de abertura e fechamento das válvulas antigotas novas e usadas, desvio-padrão e coeficiente de variação de fabricação - CVf para peças novas e de vazão - CVq para usadas. Opening and closing pressure of the leak-preventing device new and used, standard deviation, manufacturing coefficient variation - CVf for used devices.

\begin{tabular}{|c|c|c|c|c|c|c|c|c|}
\hline \multirow{5}{*}{$\begin{array}{c}\text { Válvula } \\
\mathrm{N}^{\mathrm{o}}\end{array}$} & \multicolumn{8}{|c|}{ Pressões (kPa) } \\
\hline & \multicolumn{2}{|c|}{$\begin{array}{l}\text { Modular de Baixa } \\
\text { Pressão, Nova }\end{array}$} & \multicolumn{2}{|c|}{$\begin{array}{l}\text { Modular de Baixa } \\
\text { Pressão, Usada }\end{array}$} & \multicolumn{2}{|c|}{$\begin{array}{l}\text { Modular de Alta } \\
\text { Pressão, Nova }\end{array}$} & \multicolumn{2}{|c|}{$\begin{array}{l}\text { Hadar de Baixa } \\
\text { Pressão, Nova }\end{array}$} \\
\hline & Abre & Fecha & Abre & Fecha & Abre & Fecha & Abre & Fecha \\
\hline & \multicolumn{2}{|c|}{ Pressão fabricante } & & & \multicolumn{2}{|c|}{ Pressão fabricante } & \multicolumn{2}{|c|}{ Pressão fabricante } \\
\hline & $200 \mathrm{kPa}$ & $100 \mathrm{kPa}$ & - & - & $350 \mathrm{kPa}$ & $200 \mathrm{kPa}$ & $130 \mathrm{kPa}$ & $90 \mathrm{kPa}$ \\
\hline 1 & 123,10 & 112,90 & 114,70 & 95,60 & 267,50 & 258,00 & 89,30 & 47,90 \\
\hline 2 & 114,40 & 103,40 & 101,10 & 99,80 & 235,50 & 232,60 & 91,70 & 52,60 \\
\hline 3 & 110,30 & 104,00 & 116,30 & 111,60 & 175,00 & 167,90 & 82,50 & 48,40 \\
\hline 4 & 125,70 & 106,10 & 103,70 & 93,70 & 260,40 & 251,50 & 82,20 & 48,40 \\
\hline 5 & 118,40 & 111,00 & 117,10 & 109,50 & 280,30 & 274,50 & 84,10 & 46,60 \\
\hline 6 & 115,80 & 110,80 & 124,10 & 116,00 & 290,20 & 279,00 & 95,10 & 45,00 \\
\hline 7 & 110,80 & 102,90 & 101,10 & 93,50 & 0,00 & 0,00 & 100,00 & 48,90 \\
\hline 8 & 117,30 & 106,30 & 120,70 & 98,20 & 149,80 & 139,60 & 100,80 & 51,30 \\
\hline 9 & 124,70 & 112,10 & 125,20 & 119,40 & 0,00 & 0,00 & 107,60 & 57,80 \\
\hline 10 & 124,40 & 107,60 & 123,40 & 114,70 & 331,90 & 317,20 & 95,30 & 53,90 \\
\hline 11 & 125,20 & 113,70 & 119,40 & 105,80 & 243,90 & 237,30 & 95,30 & 51,60 \\
\hline 12 & 132,80 & 116,80 & 116,80 & 107,10 & 214,00 & 210,10 & 96,10 & 51,60 \\
\hline 13 & 130,40 & 120,20 & 114,70 & 98,20 & 287,60 & 270,90 & 95,80 & 50,30 \\
\hline 14 & 113,40 & 104,20 & 105,00 & 99,50 & 339,20 & 310,40 & 100,60 & 50,30 \\
\hline 15 & 113,10 & 108,20 & 102,40 & 95,30 & 315,70 & 308,80 & 96,90 & 50,30 \\
\hline 16 & 121,00 & 112,30 & 69,90 & 50,80 & 281,90 & 274,80 & 96,90 & 50,30 \\
\hline 17 & 114,70 & 103,20 & 113,90 & 104,80 & 0,00 & 0,00 & 91,10 & 46,10 \\
\hline 18 & 118,60 & 103,40 & 108,20 & 102,10 & 273,00 & 265,90 & 97,20 & 46,10 \\
\hline 19 & 124,10 & 116,30 & 137,50 & 129,90 & 289,70 & 269,80 & 103,20 & 52,10 \\
\hline 20 & 131,70 & 114,40 & 5,20 & 4,70 & 276,90 & 265,60 & 101,30 & 46,10 \\
\hline Média (kPa) & 120,50 & 109,49 & 107,02 & 97,51 & 265,44 & 254,94 & 95,15 & 49,78 \\
\hline Desvpad (kPa) & 6,85 & 5,28 & 27,59 & 26,74 & 50,21 & 47,20 & 6,75 & 3,13 \\
\hline $\operatorname{CVf}(\%)$ & 5,68 & 4,82 & - & - & 18,92 & 18,51 & 7,10 & 6,29 \\
\hline $\mathrm{CVq}(\%)$ & - & - & 25,78 & 27,42 & - & - & - & - \\
\hline
\end{tabular}

Desvpad: desvio-padrão; CVf: coeficiente de variação de fabricação e CVq: coeficiente de variação de vazão

A válvula antigotas Hadar de baixa pressão nova apresentou pressão de abertura média de 95,15 kPa e CVf de 7,10\%, considerado marginal pela ASAE (1994). Já na pressão de fechamento, apresentou média de 49,70 kPa e CVf de 6,29\%, considerado mediano pela ASAE (1994) e bom pela ABNT (1986), consideravelmente inferior aos dados fornecidos pelo seu fabricante, onde a pressão de abertura deveria ser de $130 \mathrm{kPa}$ e a de fechamento de $90 \mathrm{kPa}$. Dentre as válvulas avaliadas, essa foi a que respondeu mais rapidamente à liberação de passagem de água.

\section{CONCLUSÕES}

O CVf e CVq do conjunto microaspersor e válvula antigotas e somente o microaspersor do grupo modular foram classificados como ruins ou inaceitáveis. A válvula antigotas Hadar foi a que apresentou o melhor desempenho em todas as pressões de serviço.

As válvulas avaliadas abriram em pressões inferiores às indicadas pelos seus fabricantes. $\mathrm{O}$ tempo de uso interferiu no desempenho das válvulas antigotas modular de baixa pressão, onde o modelo novo apresentou menor valor do CVf para a pressão de abertura e fechamento. 


\section{REFERÊNCIAS}

ABNT. ASSOCIAÇÃO BRASILEIRA DE NORMAS TÉCNICAS. Emissores para sistema de irrigação localizada: avaliação de características operacionais. Método de ensaio: Rio de Janeiro, 1986. 6 p. Projeto de norma 12:02.08.021.

ASAE. AMERICAN SOCIETY OF AGRICULTURAL ENGINEERS. Design and installation of microirrigation system. St. Joseph: ASAE EP405.1, 1994. p. 724-727.

BERNARDO, S.; SOARES, A.A.; MANTOVANI, E.C. Manual de irrigação. 7.ed. Viçosa - MG: UFV, 2005. $611 \mathrm{p}$.

COSTA, E.; LEAL, P.A.M.; CARMO JÚNIOR, R.R. Modelo de simulação da temperatura e umidade relativa do ar no interior de estufa plástica. Engenharia Agrícola, Jaboticabal, v.24, n.1, p.57-67, jan./abr. 2004.

DANTAS NETO, J.; MEDEIROS M.G.A.; AZEVEDO, C. A.V.; AZEVEDO H.M. Performance hidráulica e perfil de distribuição de água do microaspersor Naan 7110, sob diferentes condições de vento. Revista Brasileira de Engenharia Agrícola e Ambiental, Campina Grande, v.1, p.57-61, 1997.

KELLER, L.; KARMELLI, D. Trickle irrigation design parameters. Transactions of the ASAE, St. Joseph, v.17, n.2. p.668-684, 1984.

MATOS, J.A.; RAGOSO, C.R.A. Caracterização da performance hidráulica de dois emissores tipo microaspersor. Revisa Brasileira de Engenharia Agrícola e Ambiental, Campina Grande, v.1, p.1720, 1997.

MAZZER, H.R.; VILAS BOAS, M.A.; SAMPAIO, S.C. Comparação de dois métodos de ensaios radiais para microaspersores. In: CONGRESSO BRASILEIRO DE ENGENHARIA AGRÍCOLA, 32., 2003. Goiânia. Anais... Goiânia: Associação Brasileira de Engenharia Agrícola, 2003. 1 CDROM.

SAMPAIO, S.C. Uniformidade de aplicação de água por microaspersores operando em posição invertida. Ciência e Agrotecnologia, Lavras, v.25, n.6, n.1.359, nov./dez. 2001.

SANTIAGO, F.S.; MONTENEGRO, A.A.A.; MONTENEGRO, S.M.G.L. Avaliação de parâmetros hidráulicos e manejo da irrigação por microaspersão em área de assentamento. Engenharia Agrícola, Jaboticabal, v.24, n.3, p.632-643, 2004.

SAPIR, E.; SNEH, N. Riego por aspersion. Tel Aviv: MASHAV-CINADCO, 2002. 127 p.

SILVA, R.A.; SILVA, A.M. Avaliação das características hidráulicas do microdifusor autocompesante Dan Jet 7200. Ciência e Agrotecnologia, Lavras, v.27, n.4, p.873-878, 2003.

SIQUEIRA, E.C.; BARROS JÚNIOR, G. Determinação do coeficiente de variação e vazão do microaspersor Carburundum MS V versus pressão. In: CONGRESSO BRASILEIRO DE ENGENHARIA AGRÍCOLA, 32., 2003. Anais... Goiânia: Associação Brasileira de Engenharia Agrícola, 2003. 1 CD-ROM. 